\title{
A Facelift for a Two-Year-Old: Alternative Use of Facelift Approach, to Achieve Serial Excision of a Medium Sized Congenital Naevus, in a Two-Year-Old Patient
}

\author{
Marco Malahias, Diaa Othman, George Spyrou \\ Plastic Surgery Department, Pinderfields Hospital, Wakefield, UK \\ E-mail: diaa.othman@doctors.org.uk \\ Received June 30, 2011; revised September 22, 2011; accepted October 29, 2011
}

\begin{abstract}
Historically, face-lifting consisted of elevating the skin, placing it under tension to reduce the wrinkles, resecting the skin needed to accomplish this, and then securing the resected edges. However, over time, facelift surgery evolved beyond the Dorland's definition of "rhytidectomy" (excision of skin for the elimination of wrinkles [1]) and various different techniques and indications have been described. We report of the preauricular facelift approach, as described by Skoog [2] for alternative use: The excision of a giant cell tumour, utilizing this facelift technique to achieve serial excision from a two year old, male patient's face. This has resulted in a satisfactory aesthetic outcome and scar alignment in favourable area as well as excision of a significant part of the lesion, and awaiting further serial excision for completion.
\end{abstract}

Keywords: Facelift, Congenital Naevus, Facial Reconstruction

\section{Purpose}

A two-year-old male patient was born with a giant naevus involving most of the left side of his face (Figure 1). The lesion had increased both in size and pigmentation as he grew older, which prompted the parents to seek medical advice, resulting in their referral to our unit. The lesion's diameter measured $(6 \mathrm{~cm} \times 7.5 \mathrm{~cm})$. The main concern with this patient was the potential risk of malignant transformation and the overall cosmetic appearance of this young child, which may have led to him being bullied by his peers, once he reached school age.

\section{Method}

Several management options where considered including Laser therapy, dermabrasion and excision followed by sheet grafting, tissue expansion and serial excision. Both dermabrasion and laser therapy where not feasible, due to the lack penetration to deeper tissues. Excision and sheet grafting was an unacceptable solution for the parents, who rejected the idea of visible scars in their son's face.

The idea of tissue expansion was abandoned, due to the lack of sufficient, unaffected tissue between the angle of the mouth and the most anterior border of the pig- mented area. Therefore, after meticulous planning and in depth discussions with the parents, we decided on the option of serial excision of the lesion and chose the preauricular facelift approach, traditionally associated with rhytidectomy in older patients usually beyond their fifth decade of life.

The skin of the left side of the face was incised via a standard pre-auricular approach; a single dose of intravenous antibiotic (cefuroxime) was administered at induction. The skin with the overlying giant naevus was dissected mobilised and elevated (Figure 2). The intra operative findings included: 1) abnormally pigmentedskin, which was of variable thickness, resulting in areas that were easily perforated by the instruments routinely utilized for skin retraction; 2) subcutaneous fat atrophy; 3 ) an increase in tissue elasticity allowing easy stretch of the pigmented skin to achieve maximal excision, without distorting the angle of the mouth or altering the position of the ala of the nose.

\section{Result}

During the first of a total of three planned procedures $40 \%$ of the initial lesion was excised (Figures 3 and 4 ). Skin closure, without excessive tension was achieved 


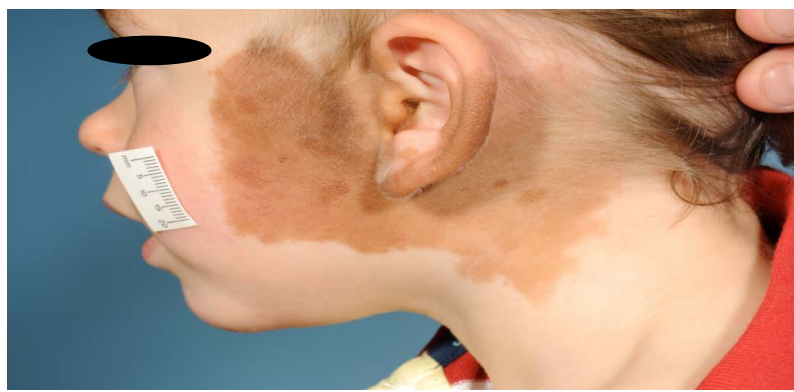

Figure 1. Preoperative lesion.

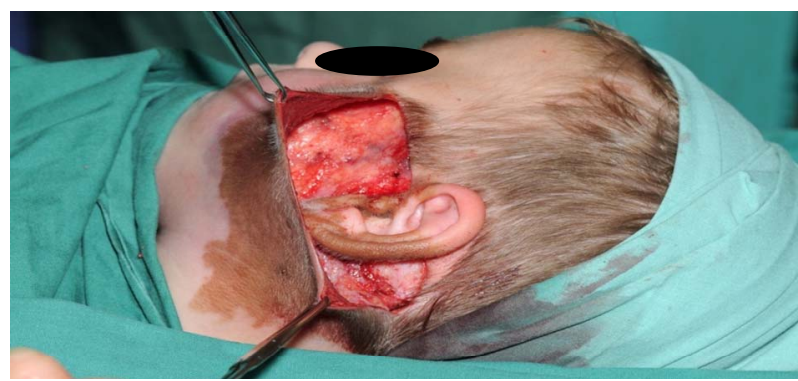

Figure 2. Intraoperative dissection.

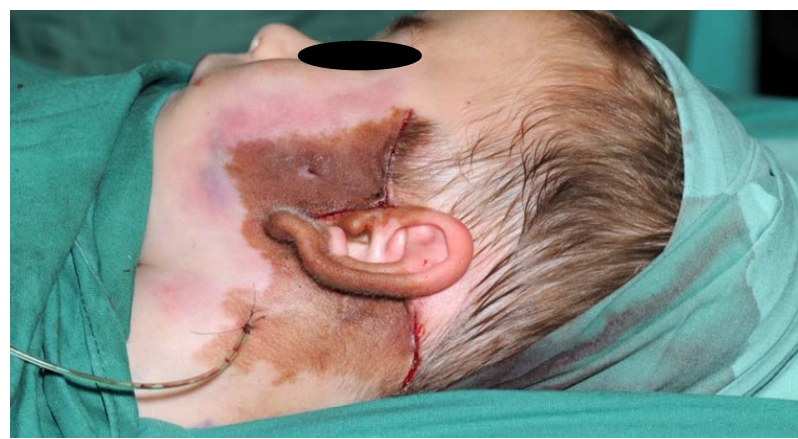

Figure 3. Immediate postoperative closure.

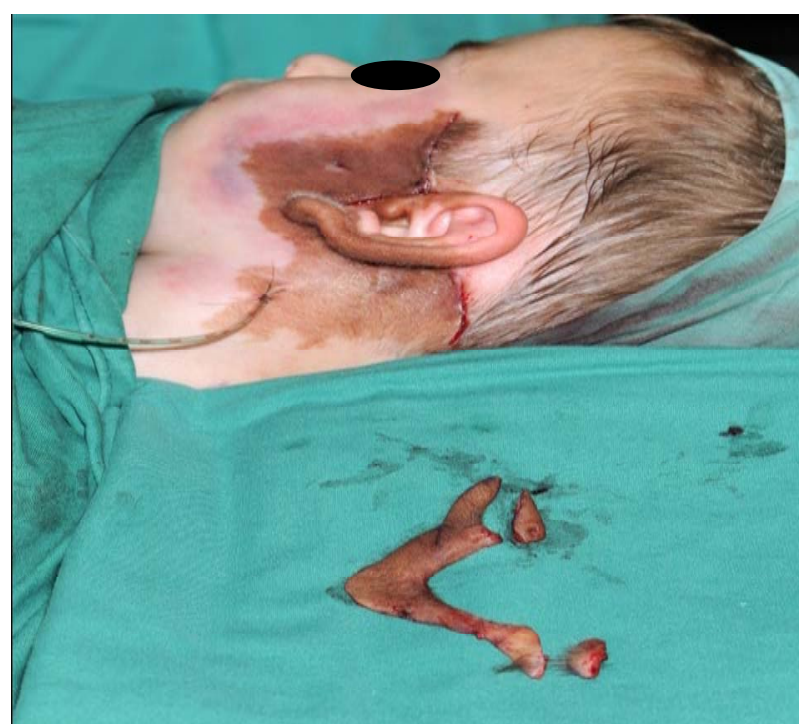

Figure 4. Immediate postoperative closure with area excised. and a small suction drain was placed in situ for 24 hours, to collect any excessive ooze from the wound. The subsequent stages where planned six to nine months apart.

\section{Discussion}

Congenital nevi are present at birth and result from a proliferation of benign melanocytes in the dermis, epidermis, or both. Occasionally, nevi that are not present at birth but are histologically identical to congenital nevi may develop during the first two years of life. This is referred to as congenital nevus tardive [1].

The aetiology from the congenital melanocytic naevi remains unclear. The melanocytes of the skin originate in the neuroectoderm, although the specific cell type from which they derive remains unknown [2-4].

Congenital nevi have been stratified into 3 groups according to size. Small nevi are less than $1.5 \mathrm{~cm}$ in greatest diameter, medium nevi are 1.5 - $19.9 \mathrm{~cm}$ in greatest diameter, and large or giant nevi are greater than $20 \mathrm{~cm}$ in greatest diameter. Giant nevi are often surrounded by several smaller satellite nevi. For giant congenital melanocytic nevi, the risk of developing melanoma has been reported to be as high as $5 \%-7 \%$ by age 60 years $[5,6]$.

Because of the increased risk of melanoma associated with congenital nevi, attempts have been made to distinguish congenital nevi from acquired nevi on the basis of histology. Distinguishing histologic features include: 1) involvement by nevus cells of deep dermal appendages and neurovascular structures (including hair follicles, sebaceous glands, arrector pili muscles, and within blood vessels walls); 2) extension of nevus cells to deep dermis and subcutaneous fat; 3) infiltration of nevus cells between collagen bundles; and 4) a nevus cell-poor sub epidermal zone [7-9].

\section{Conclusions}

Surgical removal of congenital melanocytic nevi is performed for two main reasons, first, to improve the cosmetic appearance of the patient and second, to reduce the likelihood of malignant transformation. The use of a facelift approach to solve this predicament shows how versatile surgical skills need to be, in order to find an answer to any operative challenge.

\section{References}

[1] D. Wan, "Dorland's Illustrated Medical Dictionary,” 27th Edition, WB Saunders Co., Philadelphia, 1988, p. 1464.

[2] T. Skoog and J. Ellenberg, "Skoog Technique of Facelift,” Aesthetic Plastic Surgery, 1989, p. 721, 723.

[3] O. J. Clemmensen and S. Kroon, "The Histology of 'Congenital Features’ in Early Acquired Melanocytic Nevi,” 
Journal of the American Academy of Dermatology, Vol. 19, No. 4, 1988, pp. 742-746.

doi:10.1016/S0190-9622(88)70231-5

[4] H. Ansarin, R. Soltani-Arabshahi, D. Mehregan, N. Shayanfar and P. Soltanzadeh, "Giant Congenital Melanocytic Nevus with Neurofibroma-Like Changes and Spina Bifida Occulta,” International Journal of Dermatology, Vol. 45, No. 11, 2006, pp. 1347-1350. doi:10.1111/j.1365-4632.2006.02776.x

[5] M. A. Cruz, E. S. Cho, R. A. Schwartz and C. K. Janniger, "Congenital Neurocutaneousmelanosis," Cutis, Vol. 60, No. 4, 1997, pp. 178-181.

[6] R. Silfen, P. J. Skoll and D. A. Hudson, "Congenital Giant Hairy Nevi and Neurofibromatosis: The Significance of Their Common Origin," Plastic and Reconstructive Surgery, Vol. 110, No. 5, 2002, pp. 1364-1365. doi:10.1097/00006534-200210000-00031

[7] A. R. Rhodes, et al., "Nonepidermal Origin of Malignant Melanoma Associated with a Giant Congenital Nevocellular Nevus," Plastic and Reconstructive Surgery, Vol. 67, No. 6, 1981, pp. 782-790. doi:10.1097/00006534-198106000-00012

[8] B. J. Bett, "Large or Multiple Congenital Melanocytic Nevi: Occurrence of Cutaneous Melanoma in 1008 Persons," Journal of the American Academy of Dermatology, Vol. 52, No. 5, 2005, pp. 793-797. doi:10.1016/j.jaad.2005.02.024

[9] G. J. Mark, et al., "Congenital Melanocytic Nevi of the Small and Garment Type. Clinical, Histologic, and Ultrastructural Studies,” Human Pathology, Vol. 4, No. 3, 1973, pp. 395-418. doi:10.1016/S0046-8177(73)80101-7 